\title{
Optimization of Louver Configuration in a Closed Generator
}

\author{
${ }^{1}$ Naveen kumar A., ${ }^{2}$ Dr. Rajendran \\ ${ }^{1}$ Me-Thermal Engineering RVS College of Engineering and Technology Coimbatore, India \\ ${ }^{2}$ Dean, Mechanical department RVS College of Engineering and Technology Coimbatore, India
}

\begin{abstract}
Nowadays Diesel generator assemblies become an integral part of almost all the industries, large housing units and commercial shops as well. Keeping these units in a canopy with louvers will reduce the noise pollution and at the same time thermal behavior of this system becomes a challenging task. In this project a novel approach is taken using CFD, to understand the flow and thermal behavior of the DG assembly in turn trails are done to optimize the system by changing the position of the louvers critical interpretation of the CFD results such as contour plots, velocity vectors, path and stream lines. Arriving at the optimal flow and thermal behavior inside the DG Shelter system by optimizing the following variables such as Dimensions of the baffles in the louvers, Positions of the baffles in these louvers, Orientation of the baffles in these louvers.
\end{abstract}

\section{Introduction}

A diesel generator is the combination of a diesel engine with an electrical generator (often an alternator) to generate electrical energy. Diesel generating sets are used in places without connection to the power grid, as emergency power-supply if the grid fails, as well as for more complex applications such as peak-lopping, grid support and export to the power grid. Sizing of diesel generators is critical to avoid low-load or a shortage of power and is complicated by modern electronics, specifically non-linear loads. Set sizes range from 8 to $30 \mathrm{~kW}$ (also 8 to $30 \mathrm{kVA}$ single phase) for homes, small shops \& offices with the larger industrial generators from 8 $\mathrm{kW}(11 \mathrm{kVA})$ up to $2,000 \mathrm{~kW}(2,500 \mathrm{kVA}$ three phase) used for large office complexes, factories. A $2,000 \mathrm{~kW}$ set can be housed in a $40 \mathrm{ft}(12 \mathrm{~m})$ ISO container with fuel tank, controls, power distribution equipment and all other equipment needed to operate as a standalone power station or as a standby backup to grid power. These units, referred to as power modules are gensets on large triple axle trailers weighing 85,000 pounds $(38,555 \mathrm{~kg})$ or more. A combination of these modules are used for small power stations and these may use from one to 20 units per power section and These sections can be combined to involve hundreds of power modules. In these larger sizes the power module (engine and generator) are brought to site on trailers separately and are connected together with large cables and a control cable to form a complete synchronized power plant.

\subsection{Generator size}

Generating sets are selected based on the Electrical load they are intended to supply, the electrical loads total characteristics (kWe, kVA, var's and Harmonic Content including starting currents (normally from motors) and non-linear loads. The expected duty, for example, emergency, prime or continuous power as well as environmental conditions such as altitude, temperature and emissions regulations must be taken into account as well. Most of the larger generator set manufacturers offer software that will perform the complicated sizing calculations by simply inputting site conditions and connected electrical load characteristics.

\subsection{Rating}

Generators must provide the anticipated power required reliably and without damage and this is achieved by the manufacturer giving one or more ratings to a specific generator set model. When running, the standby generator may be operated with a specified - e.g. $10 \%$ overload that can be tolerated for the expected short running time.

\subsection{Diesel engine}

\section{Components of Diesel Generator}

A diesel engine (also known as a compression-ignition engine) is an internal combustion engine that uses the heat of compression to initiate ignition to burn the fuel, which is injected into the combustion chamber. This is in contrast to spark-ignition engines such as a petrol engine (gasoline engine) or gas engine (using a gaseous fuel as opposed to gasoline), which uses a spark plug toignite an air-fuel mixture. The engine was developed by Rudolf Diesel in 1893. The diesel engine has the highest thermal efficiency of any regular internal or external combustion engine due to its very high compression ratio. Low-speed diesel engines (as used in ships and other applications where overall engine weight is relatively unimportant) can have a thermal efficiency that exceeds $50 \%$. Diesel engines are manufactured in two-stroke and four-stroke versions. 


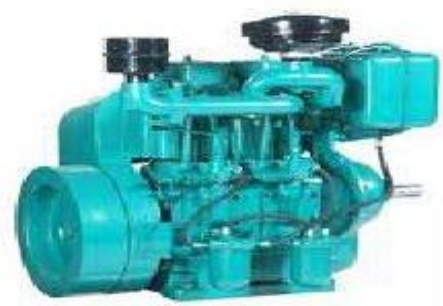

Fig 2.1 Diesel Engine

They were originally used as a more efficient replacement for stationary steam engines. Since the $1910 \mathrm{~s}$ they have been used in submarines and ships. Use in locomotives, trucks, heavy equipment and electric generating plants followed later. In the 1930s, they slowly began to be used in a few automobiles. Since the 1970 s, the use of diesel engines in larger on-road and off-road vehicles in the USA increased. As of 2007, about $50 \%$ of all new car sales in Europe are diesel.

\subsection{Alternator}

An alternator is an electromechanical device that converts mechanical energy to electrical energy in the form of alternating current. In principle, any AC electrical generator can be called an alternator, but usually the term refers to small rotating machines driven by automotive and other internal combustion engines. An alternator that uses a permanent magnet for its magnetic field is called a magneto. Alternators in power stations driven by steam turbines are called turbo-alternators.

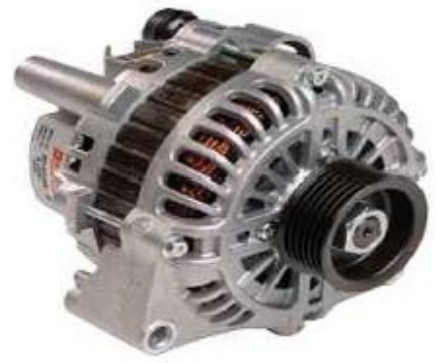

2.2 Alternator

\subsection{Battery}

In electricity, a battery is a device consisting of one or more electrochemical cells that convert stored chemical energy into electrical energy. Since the invention of the first battery (or "voltaic pile") in 1800 by Alessandro Volta and especially since the technically improved Daniel cell in 1836, batteries have become a common power source for many household and industrial applications.

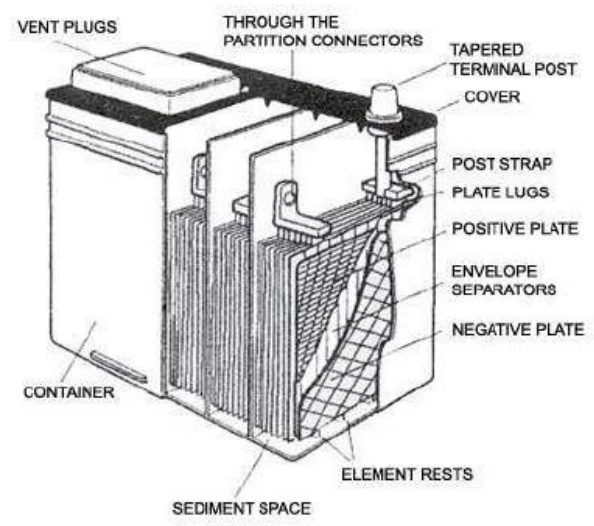

2.3. Battery

There are two types of batteries: primary batteries (disposable batteries), which are designed to be used once and discarded, and secondary batteries (rechargeable batteries), which are designed to be recharged and used multiple times. Batteries come in many size, from miniature cells used to power hearing aids and wristwatches to battery banks the size of rooms that provide standby power for telephone exchanges and computer data centers 


\subsection{Radiator (engine cooling)}

Radiators are used for cooling internal combustion engines, mainly in automobiles but also in piston-engine aircraft, railway locomotives, motorcycles, stationary generating plant or any similar use of such an engine.

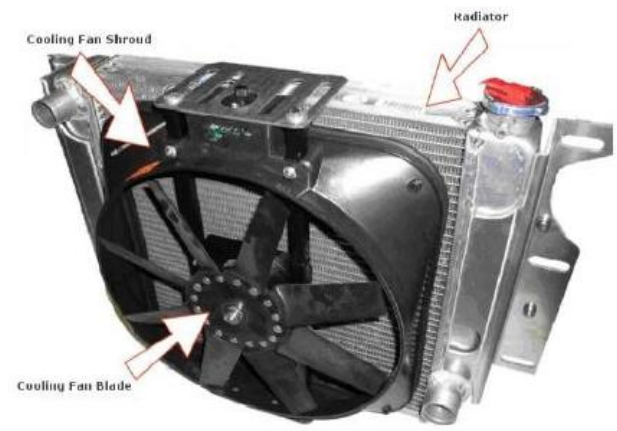

2.4. Radiator

Internal combustion engines are often cooled by passing a liquid called engine coolant through the engine block, where it is heated, then through the radiator itself where it loses heat to the atmosphere, and then back to the engine in a closed loop. Engine coolant is usually water-based, but may also be oil. It is common to employ a water pump to force the

engine coolant to circulate, and also for an axial fan to force air through the radiator.

\subsection{Canopy}

Canopy means a high cover overarching an open space. A covering, usually of cloth, suspended over a throne or bed or held aloft on poles above an eminent person or a sacred object. . A protective roof like covering, often of canvas, mounted on a frame over a walkway or door. A high overarching covering, such as the sky: "I just look up at the stars and let the vastness of that black and twinkling canopy fill my soul" (Margaret Mason). The uppermost layer in a forest is formed by the crowns of the trees. It is also called as Crown canopy. The transparent enclosure is over the cockpit of an aircraft. The part of a parachute that opens up is to catch the air.

\subsection{Sound Baffle}

A sound baffle is a construction or device which reduces the strength (level) of airborne sound. Sound baffles are a fundamental tool of noise mitigation, the practice of minimizing noise pollution or reverberation. An important type of sound baffle is the noise barrier constructed along highways to reduce sound levels at properties in the vicinity. Sound baffles are also applied to walls and ceilings in building interiors to absorb sound energy and thus lessen reverberation.

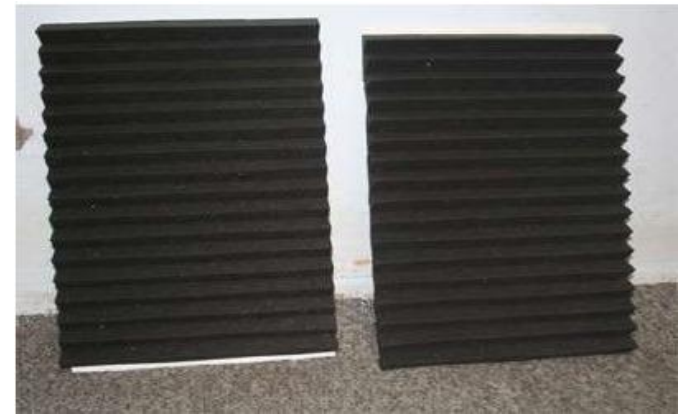

2.5. Baffle

\subsection{Louver}

A louver is a window blind or shutter with horizontal slats that are angled to admit light and air, but to keep out rain, direct sunshine, and noise. 


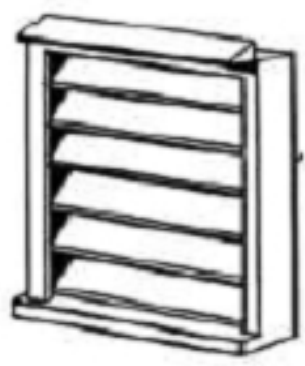

2.6.Louver

The angle of the slats may be adjustable, usually in blinds and windows, or fixed.

\subsection{Design of DG}

\section{Design and specification of DG}

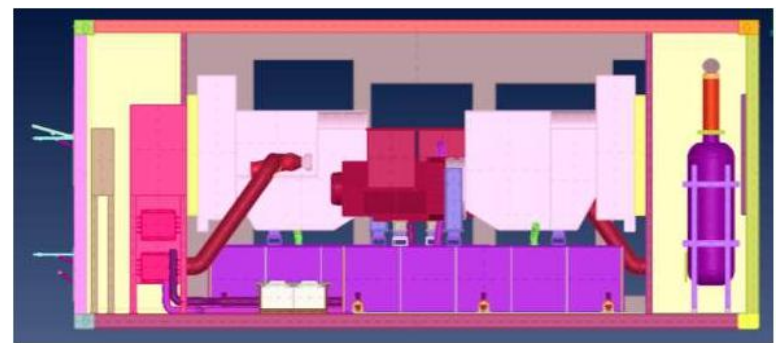

Fig. 3.1 Design of DG

\subsection{Design of DG-top view}

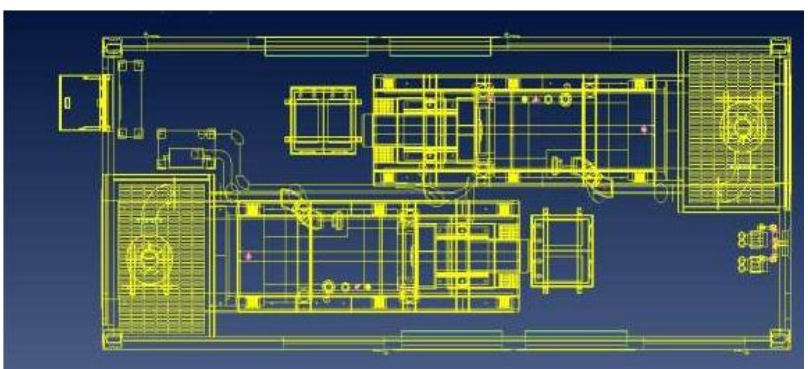

Fig. 3.2 Wire frame model

\subsection{Position of Louvers}

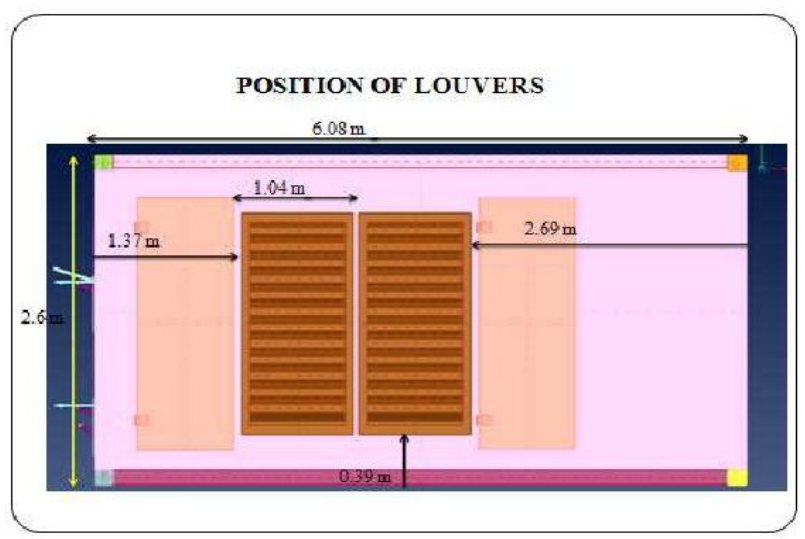

Fig. 3.3 Position of Louvers

Length of the canopy $=6.08 \mathrm{~m}$.

Height of the canopy $=2.6 \mathrm{~m}$.

Length of the inlet louvers $=1.04 \mathrm{~m}$.

Height of the inlet louvers $=1.82 \mathrm{~m}$.

Angle of baffles $=45^{\circ}$. 


\subsection{Inlet and outlet louvers arrangement:}

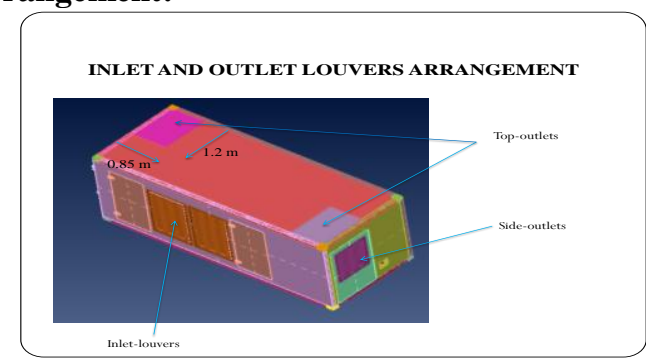

Fig 3.4 Inlet and outlet arrangement

Length of the outlet louvers $=0.85 \mathrm{~m}$.

Breadth of the outlet louvers $=1.2 \mathrm{~m}$.

No of inlet louvers $=4$.

No of outlet louvers $=4$.

\subsection{Specifications of Engine and Alternator}

\begin{tabular}{|c|c|c|}
\hline Engine & $\begin{array}{r}\text { DEUTZ- TCD2014 L6 } \\
4 \mathrm{~V}\end{array}$ & Unit \\
\hline Cooling air volume flow & 16200 & $\mathrm{~m}^{3} / \mathrm{h}$ \\
\hline $\begin{array}{c}\text { Combustion air volume } \\
\text { flow }\end{array}$ & 909 & $\mathrm{~m}^{3} / \mathrm{h}$ \\
\hline Sound power at full load & 112.1 & $\mathrm{~dB}(\mathrm{~A})$ \\
\hline Engine heat dissipation & 122.3 & $\mathrm{~kW}$ \\
\hline CAC heat dissipation & 48 & $\mathrm{~kW}$ \\
\hline $\begin{array}{c}\text { Heat } \\
\text { dissipation(convection) }\end{array}$ & 25 & $\mathrm{~kW}$ \\
\hline
\end{tabular}

\begin{tabular}{|c|c|c|}
\hline Alternator & $\begin{array}{c}\text { STAMFORD- } \\
\text { UC127AH }\end{array}$ & Unit \\
\hline $\begin{array}{c}\text { Cooling air } \\
\text { volume flow }\end{array}$ & 1850.4 & $\mathrm{~m}^{3} / \mathrm{h}$ \\
\hline Heat dissipation & 11.2 & $\mathrm{~kW}$ \\
\hline $\begin{array}{c}\text { Total air volume } \\
\text { flow } \\
\text { 1GENSET }\end{array}$ & 18959.4 & $\mathrm{~m}^{3} / \mathrm{h}$ \\
\hline $\begin{array}{c}\text { Total air volume } \\
\text { flow } \\
\text { 2 GENSETs }\end{array}$ & 37918.8 & $\mathrm{~m}^{3} / \mathrm{h}$ \\
\hline
\end{tabular}

IV. Problem definition

1) There is a marginal variation of flow rate at the various Inlets.

2) The Inlet Louvers which are closer to the Radiator Fan delivers less

3) Amount of volume of air comparing to the other one.

4) The heat generated from the engine are not effectively transferred to

5) The radiator region.

\section{Calculation}

\subsection{Mass Flow rate}

Heat generated on the engine $=122 \mathrm{kw}$.

Through convection, only $40 \%$ of heat generated on engine surface, therefore

Total heat generated on engine surface $=48.8 \mathrm{~kW}$.

$\mathrm{Q}=\mathrm{m} * \mathrm{Cp} * \Delta \mathrm{T}$. 
$\Delta \mathrm{T}=9^{0} \mathrm{c}$.

$\mathrm{Cp}=1.006 \mathrm{~kJ} / \mathrm{kg} . \mathrm{k}$

$\mathrm{Q}=48.8 \mathrm{kw}$

$48.8=\mathrm{m}^{*} 1.006 * 9$

$\mathbf{m}=5.21 \mathrm{~m}^{3} / \mathrm{s}$

\subsection{Heat Flux}

Heat generated on the engine is $\mathrm{Q}=122 \mathrm{KW}$.

Only $30 \%$ heat will be generated on the engine surface $=122 * 30 / 100$;

$\mathrm{Q}=36.6 \mathrm{KW}$

Total area of the one engine $=2.998 \mathrm{~m}^{2}$

Heat flux $=\mathrm{Q} / \mathrm{A}=36.6 / 2.998$

Heat flux on one engine surface is $=12.208 \mathrm{KW} / \mathrm{A}$.

\subsection{Introduction}

\section{COMPUTATIONAL FLUID DYNAMICS}

Fluid dynamics is a field of science which studies the physical laws governing the flow of fluids under various conditions. Great effort has gone into understanding the governing loss and the nature of fluids themselves, resulting in a complex yet theoretically strong field of research. Computational Fluid Dynamics or CFD as it is popularly known is used to generate flow simulations with the help of computer.

\section{What is computational fluid dynamics?}

Computational fluid dynamics (CFD) is the science of predicting fluid flow, Heat transfer, mass transfer, chemical reactions, and related phenomena by solving the mathematical equations which govern these processes using a numerical Process.

\subsection{History of CFD}

Computers have been used to solve fluid flow problems for many years. Numerous programs have been written to solve either specific problems, or specific classes of problems. From the mid-1970's, the complex mathematics required to generalize the algorithms began to be understood, and general purpose CFD solvers were developed. These began to appear in the early 1980's and required what were then very powerful computers, as well as an in-depth knowledge of fluid dynamics, and large amounts of time to set up simulations. Consequently, CFD was a tool used almost exclusively in research. Recent advances in computing power, together with powerful graphics and interactive 3D manipulation of models, have made the process of creating a CFD model and analyzing results much less labour intensive, reducing time and, hence, cost. As a result of these factors, Computational Fluid Dynamics is now an established industrial design tool, helping to reduce design time scales and improve processes throughout the engineering world.CFD provides a cost-effective and accurate alternative to scale model testing, with variations on the simulation being performed quickly, offering obvious advantages.

\subsection{Continuation Equation}

In fluid dynamics, the continuity equation is an expression of conservation of mass. In (vector) differential form, it is written as

$$
\frac{\partial \rho}{\partial t}+\nabla \cdot(\rho \vec{u})=0 .
$$

where $\rho$ is density, $\mathrm{t}$ is time, and $\mathrm{u}$ is fluid velocity. In Cartesian tensor notation, it is written as

$$
\frac{\partial \rho}{\partial t}+\frac{\partial}{\partial x_{j}}\left(\rho u_{j}\right)=0 .
$$

For incompressible flow, the density drops out, and the resulting equation is

in tensor form or

$$
\frac{\partial u_{j}}{\partial x_{j}}=0
$$

$$
\nabla \cdot \vec{u}=0
$$

In vector form. The left-hand side is the divergence of velocity, and it is sometimes said that an incompressible flow is divergence free. In CFD software continuity equations used to calculate the temperature distribution along the entire system. 


\subsection{Momentum Equation}

In situations in which the density is approximately constant, the flow may be termed incompressible. The Navier-Stokes equation may then be written as a

$$
\frac{\partial \vec{U}}{\partial t}+\nabla \cdot(\vec{U} \vec{U})=-\nabla p+\nabla \cdot(\nu \nabla \vec{U}) .
$$

If a turbulence model is to be employed, then the equations will change. If an eddy viscosity approach is to be used, then there are three likely modifications

The flow variables will represent average (or filtered) quantities,

The viscosity will actually be the sum of the fluid property and the calculated eddy viscosity (and will then be non constant, justifying keeping it inside the divergence operator), and assumption

The pressure will be modified to include normal-stress-like terms arising from the eddy viscosity

Thus, the equation as written will be valid for both turbulent or laminar flows, with some modification of the actual meaning of individual terms.

\subsection{Steps followed in CFD}

- Fluid domain Extraction

- $\quad$ Surface Meshing

- Volume mesh

- $\quad$ Solving the CFD problem

- Post processing

- Report generation

\subsubsection{Building Mesh}

One of the most cumbersome and time consuming part of the CFD is the mesh generation. Although for very simple flows, mesh generation is easy, it becomes very complex when the problem has many cavities and passages, Mesh generation is basically the discretization of the computational domain. The mesh in finite difference methods consists of a set of points, which are called nodes. The finite volume methods consider points that form a set of volumes which are called cells. The finite element methods used sub-volumes called elements which have nodes where the variables are defined. Values of the dependent variables, such as velocity, pressure, temperature, etc. will be described for each element.

Various forms of elements can be used. However, the most common type in CFD programs is a hexahedron with eight nodes, one at each corner, and this is known as a brick element or volume. For twodimensional applications the equivalent element is a four-nodes quadrilateral. Some finite volume programs have now been released which have the ability touse tetrahedral in three dimensions or triangles in two dimensions. Most finite element CFD codes will allow these elements to use together with a small range of other element types.

\subsubsection{Surface Meshing}

This package provides a function template to compute a triangular mesh approximating a surface. The meshing algorithm requires to know the surface to be meshed only through an oracle able to tell whether a given segment, line or ray intersects the surface or not and to compute an intersection point if any. This feature makes the package generic enough to be applied in a wide variety of situations. For instance, it can be used to mesh implicit surfaces described as the zero level set of some function. It may also be used in the field of medical imaging to mesh surfaces described as a gray level set in a three dimensional image. The meshing algorithm is based on the notion of the restricted Delaunay triangulation. Basically the algorithm computes a set of sample points on the surface, and extract an interpolating surface mesh from the three dimensional triangulation of these sample points. Points are iteratively added to the sample, as in a Delaunay refinement process, until some size and shape criteria on mesh elements are satisfied.

\subsubsection{Volumetric mesh}

Volumetric meshes are a polygonal representation of the interior volume of an object. Unlike polygon meshes which represent only the surface as polygons, volumetric meshes also discretize the interior structure of the object. One application of volumetric meshes is in finite element analysis, which may use regular or irregular volumetric meshes to compute internal stresses and forces in an object throughout the entire volume of the object. In this research, a procedure called the Reference Jacobian based Mesh Optimization has been developed for the optimization of 3D mesh quality by node repositioning. The procedure is designed to improve 
the quality or geometric shape of mesh regions and boundary mesh faces while keeping the improved mesh as close as possible to the original mesh. The quality measure optimized in the procedure is the Condition Number shape measure which quantifies the distortion of a trivalent element corner from an "ideal" corner of a canonical element. This "ideal" corner is usually considered to be formed by 3 unit vectors coincident at the origin and lying along the coordinate axes but any other definition may be used in its place. The overall procedure consists of iterations involving node repositioning on the boundary followed by node repositioning in the interior (with boundary nodes fixed). The procedure has proved to be very effective in improving mesh quality of multimaterial tetrahedral and hexahedral meshes while minimizing changes to the mesh characteristics and to the Discrete boundary surfaces.

\subsubsection{Solving the CFD problem}

1. Reading the file. The reading the file should clear as case file or data file or case and data file. In this we have to read case and data file.

2. Scaling the grid.

3. Checking the grid

4. Defining the models. Model should define whether it is steady or Unsteady and whether it is viscous. The model is defined here is steady and viscous.

5. Defining the materials.

6. Defining the boundary condition

7. Controls

8. Initialize

9. Monitor

10. Iterate

The component that solves the CFD problem is called the Solver. It produces the required results in a non-interactive/batch process. A CFD problem is solved as follows:

The partial differential equations are integrated over all the control volumes in the region of interest. This is equivalent to applying a basic conservation law (for example, for mass or momentum) to each control volume .The algebraic equations are solved iteratively. An iterative approach is required because of the nonlinear nature of the equations, and as the solution approaches the exact solution, it is said to converge.

For each iteration an error, or residual, is reported as a measure of the overall conservation of the flow properties. How close the final solution is to the exact solution depends on a number of factors, including the size and shape of the control volumes and the size of the final residuals. Complex physical processes, such as combustion and turbulence, are often modeled using empirical relationships. The approximations inherent in these models also contribute to differences between the CFD solution and the real flow.

The solution process requires no user interaction and is, therefore, usually carried out as a batch process. The solver produces a results file which is then passed to the post-processor.

\subsubsection{Post Processing}

The post-processor is the component used to analyze, visualize and present the results interactively. Post-processing includes anything from obtaining point values to complex animated sequences.

Examples of some important features of post-processors are:

1. Visualization of the geometry and control volumes.

2. Vector plots showing the direction and magnitude of the flow.

3. Visualization of the variation of scalar variables (variables Which have only magnitude, not direction, such as temperature, pressure and speed) through the domain.

4. Quantitative numerical calculations.

5. Animation.

6. Charts showing graphical plots of variables.

7. Hardcopy and online output.

\subsubsection{Report Generation}

All charts, tables, figures, and comments automatically become report content. The report component order can be adjusted and figures can be 3D Viewer files or bitmaps. Different output formats are available, including HTML. 


\subsection{Applications of CFD}

Applications of CFD are numerous

1.Flow and heat transfer in industrial processes (boilers, heat exchangers, combustion equipment, pumps, blower, piping, etc.).

2. Aerodynamics of ground vehicles, aircraft, missiles.

3. Film coating, thermoforming in material processing applications.

4. Flow and heat transfer in propulsion and power generation systems.

5. Verification, heating, and cooling flows in buildings.

6. Chemical vapor deposition (CVD) for integrated circuit manufacturing.

7. Heat transfer for electronics packaging applications.

\subsection{Advantages of CFD}

\subsubsection{Relatively low cost}

1. Using physical experiments and tests essential engineering data for design can be expensive.

2. CFD simulations are relatively inexpensive, and costs are likely to decrease as computers become more powerful.

6.6.2 SPEED.

1. CFD simulations can be executed in a short period of time.

2. Quick turnaround means engineering data can be introduced early in the design process.

\subsubsection{Ability to simulate Ideal conditions.}

1. CFD allows great control over the physical process, and provides the ability to isolate specific phenomena for study.

2. Example: a heat transfer process can be idealized with adiabatic, constant heat flux, or constant temperature boundaries.

\subsubsection{Comprehensive Information}

1. Experiments only permit data to be extracted at a limited number locations In the system (e.g. pressure and temperature probes, heat flux gauges, etc).

2. CFD allows the analyst to examine a large number of locations

in the region of interest, and yields a comprehensive set of flow parameters for examination.

\subsection{Limitations of CFD}

\subsubsection{Physical models}

1. CFD solutions rely upon physical models of real world processes.

2. The CFD solutions can only be as accurate as the physical models on which they are based.

\subsubsection{Numerical errors}

1. Solving equations on a computer invariably introduces numerical errors.

2. Round-off error; due to finite word size available on the computer, Round-off errors will always exist ( though they can be small in most cases).Truncation error: due to approximate in the numerical models. Truncation errors will go to zero as the grid is refined. Mesh refinement is one way to deal with truncation error.

\subsection{Boundary conditions}

The governing equation of fluid motion may result in a solution when the boundary conditions and the initial conditions of specified. The form of boundary conditions that is required by any partial differential equation depends on the equation itself and the way that it has been discredited.

Common boundary conditions are classified either in terms of the numerical value that have to be set or in terms of the physical type of boundary condition. The physical boundary conditions that are the commonly observed in the fluid problems are as follows:

\subsubsection{Solid walls}

Many boundaries within the fluid flow domain will be solid walls, and these can be either stationary or moving walls. If the flow is laminar then the velocity components can be set to be the velocity of walls. When the flow is turbulent, however, the situation is more complex. 


\subsubsection{Inlets}

At an inlet, fluid enters the domain and therefore, its fluid velocity or pressure or the mass flow rate may be known. Also, the fluid may have certain characteristics, such as turbulence characterizes which need to specified.

\subsubsection{Symmetry Boundaries}

When the flow is symmetrical about some plane there is no flow through the boundary and the derivatives of the variables normal to the boundary are zero.

\subsubsection{Cyclic or Periodic boundaries}

These boundaries come in pairs and are used to specify the flow has the same values of the variables at equivalent position and both of the boundaries.

\subsubsection{Pressure Boundary Conditions}

The ability to specify a pressure condition at one or more boundaries of a computational region is an important and useful computational tool. Pressure boundaries represent such things as confined reservoirs of fluid, ambient laboratory conditions and applied pressures arising from mechanical devices. Generally, pressure condition cannot be used at boundary where velocities are also specified, because velocities are influenced by pressure gradients. The only exception is when pressures are necessary to specify the fluid properties. E.g., density crossing a boundary conditions boundary so that the velocity at the boundary is zero. Since the static pressure condition says nothing about fluid outside the boundary (i.e., other than it is supposed to be the same as the velocity inside the boundary) it is less specific than the stagnation pressure condition. In this sense the stagnation pressure condition is generally more physical and is recommended for most applications.

\subsubsection{Outflow boundary conditions}

In many simulations there is need to have fluid flow out of one or more boundaries of the computational region.

In compressible flow, when the flow speed at the outflow boundary is supersonic, it makes little difference how the boundary conditions are specified since flow disturbances cannot propagate upstream. In low speed and incompressible flows, however, disturbances introduced at an outflow boundary can have an effect on the entire computational region.

The simplest and most commonly used outflow condition is that of "continuative "boundary. Continuative boundary conditions consist of zero normal derivate at the boundary for all quantities. The zeroderivative condition is intended to represent a smooth continuation of the flow through the boundary. As a general rule, a physically meaningful boundary condition such as a specified pressure condition should be used at out flow boundaries, whenever possible.

When a continuative condition is used it should be placed as far from the main flow region as is practical so that any influence on the main flow will be minimal.

\subsubsection{Opening Boundary Condition}

If the fluid flow crosses the boundary surface in either direction an opening boundary conditions needs to be utilized. All of the fluid might flow out of the domain, or into the domain, or a combination of the two might happen.

\subsubsection{Free Surfaces and Interface}

If the fluid has a free surface, then the surface tension forces need to be considered. This requires utilization of the Laplace's equation which specifies the surface tension-induced jump in the normal stress $\mathrm{p} s$ across the interface.

\subsection{Boundary Condition for this Project}

1. Energy dissipation from Engine, CAC and through convection are modeled with $122.3 \mathrm{Kw}, 48 \mathrm{Kw}$, and $25 \mathrm{Kw}$ respectively.

2. In thermal boundary condition the internal emissivity is $\mathbf{0 . 6 5}$.

3. Inlets are assumed to be pressure inlet at total pressure $=0$.

4. Outlets are assumed to be pressure outlet at static pressure $=0$.

5 . Fans are designed by fans boundary condition and it produce the mass flow rate of $5.21 \mathrm{~m}^{3} / \mathrm{s}$.

6 . Engine walls are assumed to be heat generating source.

7. Rest of the walls is assumed to be adiabatic walls

8. Fan boundary condition is setup as fan performance curve (Q vs. $\Delta \mathrm{p}$ ) 


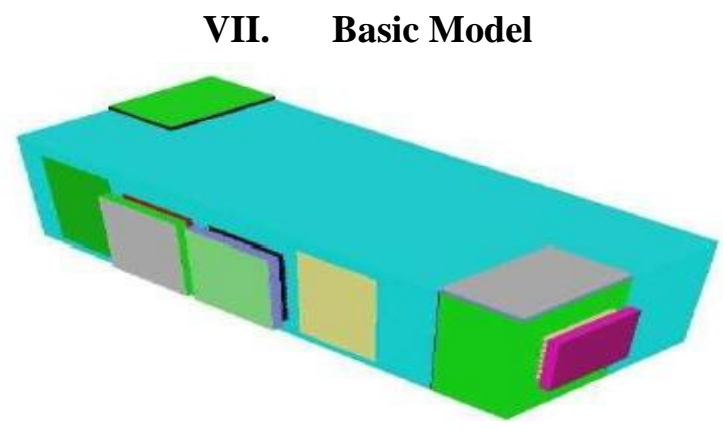

Fig. 7.1 Design of base model

The position of louvers which are close by is shown in the figure 7.1

\subsubsection{Wire frame Base Model Design}

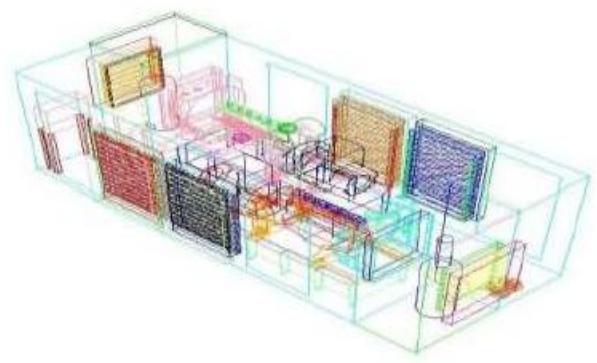

Fig. 7.2 Wire frame model for base model

\subsubsection{Surface Meshes for Base Model}

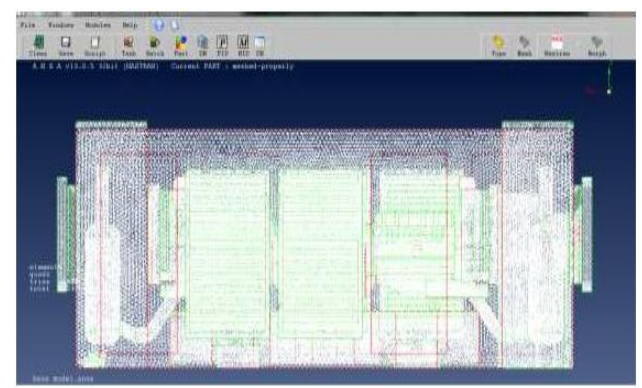

Fig. 7.3 Surface mesh for base model

The surface mesh is discretized by the triangular elements, which is because of the complexity of the geometry.

\subsubsection{Volume mesh for base model}

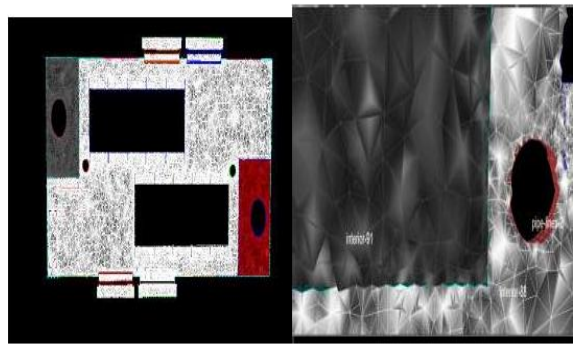

Fig. 7.4 Volume mesh for base model 


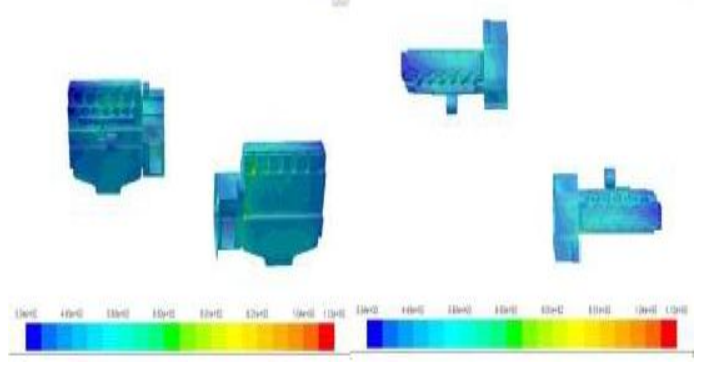

7.1.4 Meshing Details

Table 7.5 Meshing detail for base model

\begin{tabular}{|c|c|c|c|c|c|c|}
\hline Model & $\begin{array}{c}\text { Surface } \\
\text { Mesh } \\
\text { Elements }\end{array}$ & $\begin{array}{c}\text { Mesh } \\
\text { Type }\end{array}$ & Quality & $\begin{array}{c}\text { Volume } \\
\text { Mesh } \\
\text { Elements }\end{array}$ & $\begin{array}{c}\text { Mesh } \\
\text { Type }\end{array}$ & Quality \\
\hline Base & $\mathbf{5 4 8 0 5 1}$ & Tetra & $\mathbf{0 . 6}$ & $\mathbf{2 2 5 3 8 7 4}$ & Tetrahedral & $\mathbf{0 . 8 8 9}$ \\
\hline
\end{tabular}

\subsubsection{Path Lines}

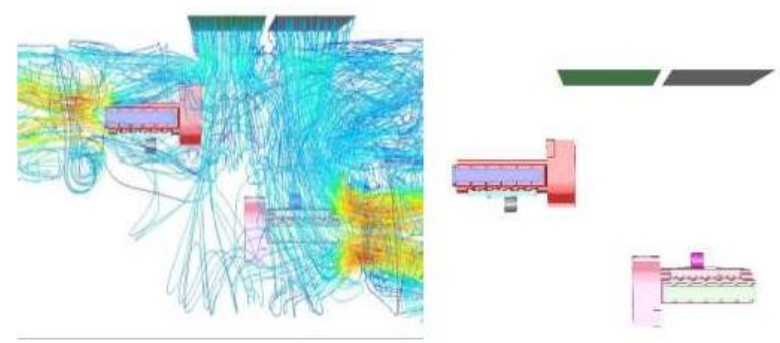

Fig. 7.6 Path lines for base model

\subsubsection{Velocity Vector}

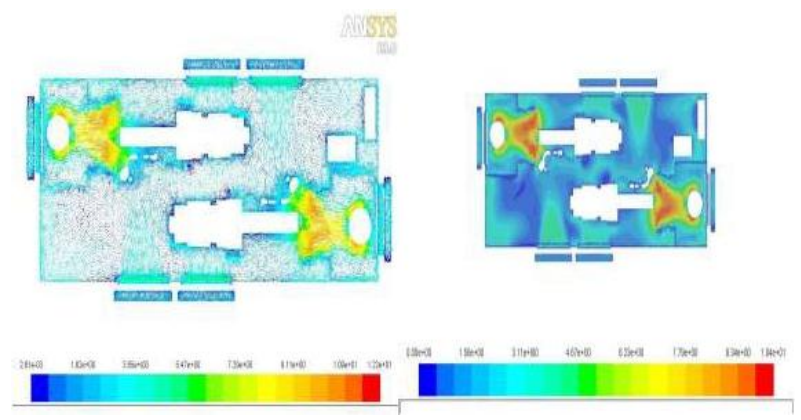

Fig. 7.7 Velocity vectors and Velocity magnitude

From the velocity vector the mass flow from the louvers is not effectively flowing on the surface of the engine which is closer by

\subsubsection{Temperature on the Engines}

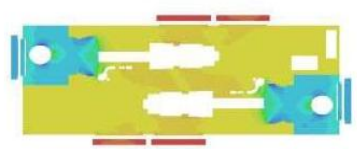

Fig. 7.8 Temperature distribution on engine surface for base model 
The engine max surface temperature will be 1053k. thus heat dissipation on the surface of the engine will be very low

\subsubsection{Pressure Distribution}

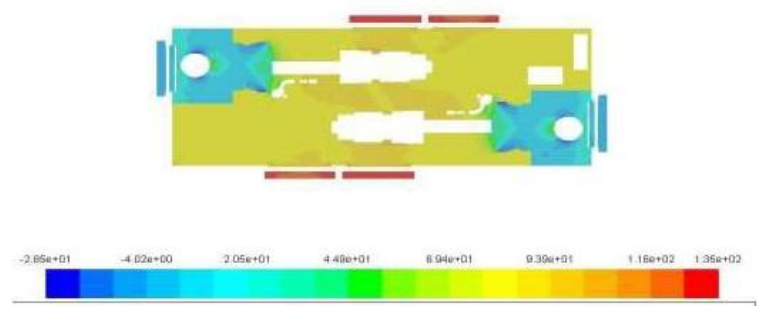

Fig. 7.9 Pressure distribution on base model

The static pressure will be higher inside the canopy except in the exhaust room.

\subsubsection{Temperature Distribution}

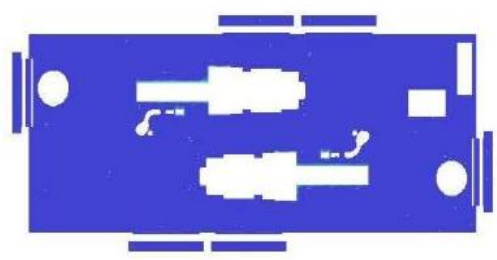

\subsubsection{Temperature Result}

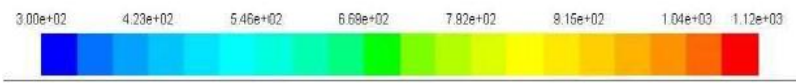

Fig. 7.10 Temperature distribution for base mode

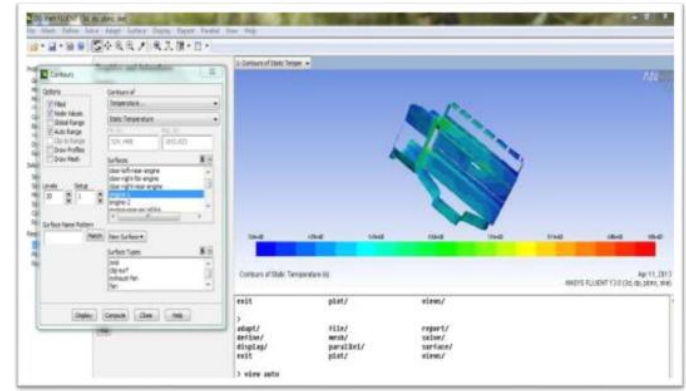

Fig. 9.11 Max and Min temperature on engine surface for base model

\section{References}

[1]. Fridriksson, H., Sund'en, B., and Hajireza, S. "A theoretical study on the heat transfer process in diesel engines". WIT Transactions on Engineering Sciences, 68, pp. 177-188, 2010

[2]. Hohenberg, G. “Advanced Approaches for Heat Transfer Calculations”. SAE Transactions, 88,pp. 61-77. SAE paper 790825, 1979.

[3]. John Anderson, text book of "introduction to computational fluid dynamics"

[4]. CFD-Wiki http://www.cfd-online.com/Wiki/Main Page

[5]. J.H. Ferziger and M. Peric, Computational Methods for Fluid Dynamics. Springer, 1996

[6]. C. Hirsch, Numerical Computation of Internal and External Flows. Vol. and II. John Wiley \& Sons, Chichester, 1990.

[7]. P. Wesseling, Principles of Computational Fluid Dynamics. Springer, 2001.

[8]. C. Cuvelier, A. Segal and A. A. van Steenhoven, Finite Element Methods and Navier-Stokes Equations. Kluwer, 1986. S. Turek, Efficient Solvers for Incompressible Flow Problems: An Algorithmic and Computational Approach, LNCSE 6, Springer, 1999.J. Donea and A. Huerta, Finite Element Methods for Flow Problems. Johniley \& Sons, 2003

[9]. S. Turek, Efficient Solvers for Incompressible Flow Problems: An Algorithmic and Computational Approach, LNCSE 6, Springer, 1999.

[10]. J. Donea and A. Huerta, Finite Element Methods for Flow Problems. Johniley \& Sons, 2003. 